\title{
Connaissances et Pratiques traditionnelles versus Conservation de la biodiversité dans les aires protégées : Cas des populations riveraines du lac Lungwe, Est RD Congo.
}

\author{
Léonard K. MUBALAMA ${ }^{1,5}$, Jean de Dieu WASSO ${ }^{3}$, Germaine BUHENDWA ${ }^{2}$, Félix IGUNZI ${ }^{2}$, \\ Bernard KANDJI ${ }^{2}$, Marcelin IBUCWA ${ }^{3}$ et Patrick KONGOLO ${ }^{3,4}$ \\ 1. Centre de Recherche en Gestion de la Biodiversité et Changement climatique (CRGBC), RD Congo/ISDR- \\ Bukavu, RD Congo \\ 2. Institut Congolais pour la Conservation de la Nature (ICCN), Réserve Naturelle d'Itombwe, RD Congo \\ 3. Africapacity Project, Bukavu, RD Congo. \\ 4. Université Evangélique en Afrique (UEA-Bukavu), RD Congo \\ 5. Académie Royale des Sciences d'Outre-Mer/Belgique
}

\begin{abstract}
RESUME
Les questions de conservation et de développement, de normes culturelles et de valorisation des ressources naturelles sont liées et ne peuvent être comprises isolément. Dans le contexte actuel du lac Lungwe, la compréhension de telles relations passe nécessairement par une meilleure connaissance des différents systèmes de gestion des ressources naturelles en place. Dans ce contexte, mieux connaître et préserver les services éco systémiques et valeurs socioculturelles du lac Lungwe dans la Réserve Naturelle d'Itombwe s'avère une nécessité pour sa valorisation. C'est d'ailleurs, l'objectif de la présente étude portant sur les croyances des communautés locales face à la gestion du lac Lungwe. L'importance et l'apport des croyances locales et traditions despeuples autochtones n'est plus a démontré dans la gestion d'une aire protégée et la valorisation des services écosystémiques. Le lac Lungwe en est un exemple concret. En effet, à l'issue des enquêtes menées au sein des villages environnants le lac Lungwe dans la chefferie de Lwindi, les résultats ont démontré que plusieurs mythes étaient jadis attribués au lac Lungwe par des ancêtres. Ces pratiques continuent à être transmises d'une génération à l'autre. Cependant, les pratiques culturelles relatives à la conservation de la biodiversité représentent, une valeur qui a été négligée ou considérée de moindre importance par les décideurs ou certains milieux scientifiques, par le simple fait qu'on les rattache à la superstition. Ces enquêtes nous ont révélé l'actuel éveil de conscience des communautés locales souhaitant la valorisation du lac Lungwe par des activités touristiques. Aussi, la réhabilitation de telles pratiques s'inscrirait dans le cadre de la stratégie nationale pour la conservation et l'utilisation durable de la diversité biologique et le partage équitable des bénéfices de l'exploitation des ressources naturelles. Cette stratégie s'appuie, entre autres, sur la prise en compte des modes traditionnels de Pratiques culturelles et conservation de la biodiversité au sein de la RNI et relative à la gestion des ressources naturelles. Ce qui s'avère, non seulement une piste de solution pour le désenclavement et développement des entités autour du lac, mais aussi, une piste réaliste par rapport au contexte du mode de gestion de la RNI qui les englobe.
\end{abstract}

Mots clés : Conservation, Croyances traditionnelles, Lac Lungwe, Réserve Naturelle d'Itombwe,

\begin{abstract}
The issues of conservation and development, cultural norms and the enhancement of natural resources are linked and cannot be understood in isolation. In the current context of Lungwe Lake, understanding such relationships necessarily requires a better understanding of the different natural resource management systems in place. In this context, better understanding and preserving the ecosystem services and socio-cultural values of Lake Lungwe in the Itombwe Nature Reserve (RNI) is a necessity for its development. This is the objective of this study on the beliefs of local communities regarding the management of Lungwe Lake. The importance and contribution of local beliefs and traditions of indigenous peoples is no longer demonstrated in the management of a protected area and the enhancement of ecosystem services. Lungwe Lake is a concrete example. Indeed, at
\end{abstract}


the end of the surveys carried out in the villages surrounding Lungwe Lake in the chiefdom of Lwindi, the results showed that several myths were once attributed to Lungwe Lake by ancestors. These practices continue to be passed down from generation to generation. However, cultural practices relating to biodiversity conservation represent a value that has been overlooked or considered less unimportant by policy makers or some scientific circles, simply because they are linked to superstition. different interpretations and or negligence on the part of certain people.

These surveys revealed to us the current awakening of consciousness of local communities wishing to enhance the value of Lungwe Lake through tourism activities. Also, the rehabilitation of such practices would be part of the national strategy for the conservation and sustainable use of biological diversity and the equitable sharing of the benefits of the exploitation of natural resources. This strategy is based, among other things, on taking into account traditional methods of cultural practices and biodiversity conservation within the RNI and relating to the management of natural resources. This turns out to be not only a possible solution for the opening up and development of entities around the lake, but also, a realistic avenue with regard to the challenge of the current mode of management of the Itombwe Natural Reserve which encompasses them.

Key words: Conservation, Traditional beliefs, Lungwe Lake, Itombwe Nature Reserve

\section{CONTEXTE ET INTRODUCTION}

\subsection{Contexte}

La protection des savoirs traditionnels en matière de biodiversité focalise une attention toujours grandissante. Les interrogations de conserve et de amplification, de normes culturels et de exploitation des ressources natures sont liées et ne savent être comprises clairement (Sow, 2003). Dans sa recherche, Condé (1997) souligne que : l'implication des populations locales est depuis une décennie à la base des stratégies et conventions environnementales, parce que les services qui ont en charge la conserve des environnements dans les pays sous-développés sont incorrigiblement inadaptés aux difficultés auxquels ils sont comparés.

Dans de nombreuses régions, la crise de la biodiversité est intérieurement mélangée avec celle des cultures indigènes. Ainsi, une part immense des zones protégées de la planète - outils capitaux pour la protection de la diversité biologique — se trouvent complètement ou imparfaitement sur des terres, des territoires et des ressources indigènes. Cet surprenant concours spatial forme à la fois une bonne occasion et un réel enjeu pour la protection de la biodiversité et le port des moyens de aliment des peuples aborigènes. L'article 10 relatif à l'utilisation durable « des éléments constitutifs de la diversité biologique » complète cette recommandation en insistant sur les droits coutumiers de ces populations. La exigence d'un partage équitable des privilèges découlant de ces « consciences, innovations et pratiques traditionnels » est également explicitement soulignée dans le Préambule.

Depuis la signature de la Convention sur la diversité biologique (CDB, 1992), les dimensions politiques et juridiques de la conservation de la biodiversité se sont affirmées. Parmi celles-ci, la exploration des savoirs traditionnels des associations autochtones et locales est devenue une interrogation centrale des tractations. Ces savoirs ont été investis d'un rôle décisif dans la protection de la biodiversité et dans l'instauration d'un marché des ressources génétiques. Ils ont été pour cela requalifiés comme patrimoine culturel à respecter, information à protéger ou encore marchandise à valoriser pour une nouvelle économie de la connaissance. L'article 8j de la $\mathrm{CDB}$ se réfère à la conservation in situ. Il tempère le droit souverain des États d'exploiter leurs propres ressources par la proposition de prendre en compte une catégorie essentielle d'acteurs : les communautés autochtones et locales. «Sous réserve des dispositions de sa législation nationale, [chaque Partie contractante] respecte, préserve et maintient les connaissances, innovations et pratiques des communautés autochtones et locales qui incarnent des modes de vie traditionnels présentant un intérêt pour la conservation et l'utilisation durable de la diversité biologique. »

L'article 2 de la CDB (emploi des termes) se garde bien de préciser les notions de associations locales et de populations indigènes, tout comme celle de pratiques traditionnels. On peut présupposer que ce sont les rapports de proximité et d'homéostasie que ces associations entretiennent avec la nature, et donc l'appréhension qu'elles en ont, qui justifient des consciences, des innovations et des pratiques traditionalistes. Si de abondantes études anthropologiques ont mis en évidence la élevée diversité des modes d'appréhension de la nature, les consciences 
touchant à ces questions restent partiels ou incomplètes, les analyses comparatives tendant à répandre la pluralité des cas regardés ou, au contraire, à confiner chaque exemple dans sa bizarrerie (Pinton \& Grenand, 2007). Lequel peut-on dire aujourd'hui de ces « savoirs traditionnels »?

Les savoirs qualifiés de traditionnels sont en réalité très divers et définissent plus un contre-type par rapport à une norme technique contemporaine. Disqualifiés depuis longtemps par la modernisation technique, ils ne sont plus conformes à un ensemble de connaissances infusant lentement au gré des étapes de la vie sociale. Leur mode de transmission est menacé par de multiples facteurs extérieurs. Les jeunes reproductions ont fréquenté l'école et sont de moins en moins les gardiens de ces savoirs, qui ont aptitude à se diviser et à s'individualiser. Autrement dit, les cadres socioculturels « traditionnels » du maintien de la diversité culturelle ont perdu de leur pertinence auprès des populations locales qui, en fonction du contexte sociopolitique, réagissent différemment aux nouveaux défis qui se posent à elles.

Pour y répondre, il nous semble constructif de nous référer à la notion de savoir local. Nous avons montré que le qualificatif de traditionnel sous-entend des situations d'exclusivité, d'identité et de profondeur historique de moins en moins aisées à saisir. Le qualificatif de local renvoie d'abord à une échelle d'observation et à un ancrage territorial. Il ne peut être confondu ni avec l'espace de la tradition, ni avec le lieu d'une civilisation vivant en harmonie avec la nature. Sachant que la philosophie de la CDB repose sur un projet d'intégration sociale des communautés traditionnelles aux nouvelles dynamiques de développement durable, ce processus de requalification peut être compris comme une « réhabilitation » du local au sein d'une société globale.

\subsection{Introduction}

La thématique des savoirs locaux et des savoirs traditionnels a été propulsée sur la scène internationale par la déclaration de Rio en 1992 qui apporte une reconnaissance des savoirs des populations locales dans la gestion de l'environnement. En 1993, la Convention sur la diversité biologique considère les savoirs traditionnels comme un patrimoine commun de l'humanité et propose un partage équitable des avantages qui découlent de la conservation et de l'utilisation durable de la diversité biologique (Carneiro da Cunha, 2012). A partir de 1980, l'importance des savoirs lieux est prise en compte dans les débats universels sur la nature (Tubiana, 2005 ; Roué, 2012). Cela a ouvert la voie aux chercheurs de diverses disciplines scientifiques qui en ont trouvé un champ d'investigation (Sow, 2003 ; Savadogo et al. 2011 ; Sene et al. 2013 ; Renard et al. 2013). L'intégration des pratiques traditionnelles dans le processus de gestion moderne des aires protégées ou de la biodiversité pourrait en constituer une stratégie efficace et durable (Ehinnou Koutchika et al. 2014).

La littérature académique a longuement débattu de la définition de la connaissance traditionnelle et ses variantes. Connaissance locale et connaissance autochtone. Les termes apparentés « connaissance écologique locale » et « connaissance traditionnelle » ont évolués en fonction des exigences des termes spécifiques pour décrire la connaissance de l'environnement naturel. Les savoirs lieux sont de plus en plus sollicités dans les allures scientifiques transdisciplinaires ou dans des exercices de gestion de la nature. L'expression "connaissances traditionnelles" englobe les connaissances, les innovations et les pratiques des groupes indigènes et locales (CDB, 1992). Il s'agit des savoirs et des savoir-faire en matière d'utilisation et de conservation de la diversité biologique et des croyances traditionnelles qui leur sont associées.

Dans cette étude le terme Connaissance Ecologique Traditionnelle se réfère à la description de Berkes (1993, Berkes et al. 2000) - 'Un ensemble de connaissances cumulatives, de pratiques et de croyances évolutifs à travers des processus adaptifs et transmis de génération en génération par la culture, sur les rapports des êtres vivants (y compris les hommes) entre eux et avec leur environnement. Par contre, la tradition, comme le montre Bonte (1992), est « ce qui d'un passé persiste dans le présent où elle est transmise et demeure agissante et acceptée par ceux qui la acquièrent et qui, à leur tour, au fil des reproductions, la donnent ». Elle peut aussi être entendue comme « un point de vue que les hommes du présent développent sur ce qui les a précédés » (Berard \&Marchenay, 2004). De fait, toutes les sociétés humaines sont traditionnelles.

Des anthropologistes et des ethnologues de la nature ont alors traversé la planète, dans le sillage des écologues et naturalistes. Leurs travaux ont permis de pourchasser l'analyse des liens compliqués entre nature et culture et de mettre en évidence l'existence des savoirs traditionnels au sein des populations vivant dans des zones particulièrement vulnérables du point de vue des menaces environnementales. En reconnaissant d'une part que 
les pratiques révèlent un processus d'adaptation, transmis entre générations, des relations entre les humains et leur environnement (Berkes et al. 2000) et d'autre part que "la légitimité du savoir est liée à son inscription dans une lignée, indissociable de la relation à un territoire » (Roué \&Nakashima 2002), les anthropologistes ont jeté les bases de la essentiel implication des populations dans la produit des savoirs éco systémiques. Même si le systématisme entre savoir traditionnel et conserve n'existe pas (Dove, 2006), cette posture conduit le caractère obligatoire de démarches participatives dans le domaine de la protection de la biodiversité contenant, parfois, pour les peuples locales, une reconfiguration de leurs commodes.

Les communautés locales et peuples autochtones se sont révélés être des gardiens responsables de leurs terres et de leurs ressources depuis des millénaires. Les cultures autochtones ont survécu et prospéré dans toutes les régions du monde en s'appuyant sur les connaissances traditionnelles et sur des pratiques de gestion durable des ressources. Néanmoins, ni les communautés autochtones, ni les sociétés industrialisées, ne sont protégées de la mondialisation de l'économie dont les répercussions mal maîtrisées mettent en péril les biens publics environnementaux qui sont d'une importance capitale pour la culture, la spiritualité et la subsistance des peuples autochtones.

Aussi, les ruraux africains ont développé dans leurs perceptions, une série de savoirs locaux protégeant leurs activités de survie, concourant par ricochet, à la conservation des forêts. Cependant, comparativement à l'Afrique de l'Ouest (Bénin, Togo, Côte d'Ivoire, Gabon, Niger, Guinée, Sénégal, Burkina Faso, etc.) où la littérature scientifique marque une présence dans cette thématique, en République Démocratique du Congo (RDC) par contre, elle accuse un certain manque, en dépit de l'existence desdites commodes au sein des associations locales. La diversité des bois sacrés, le rôle joué par les pratiques traditionnelles dans l'encadrement des pratiques locales de survie et les facteurs socioculturels influant sur leur décadence y restent faiblement documentés (Kyale \& Maindo, 2017).

Dans le contexte actuel de du lac Lungwe, la compréhension de telles relations passe nécessairement par une meilleure connaissance des différents systèmes de gestion des ressources naturelles en place. Cette implication est d'autant plus justifiée que les pratiques culturelles relatives à la conservation de la biodiversité représentent, en Afrique, une valeur qui a été négligée ou considérée sans importance par les décideurs ou certains milieux scientifiques, par le simple fait qu'on les rattache à la superstition. Omari cité par Byers (1997) observe que la révérence des Africains à l'égard de la nature et des endroits naturels était une attitude et pratique religieuse qui, tout en se développant, remplissait indirectement des fonctions sociales au sein de certaines communautés (par exemple, l'initiation des jeunes au passage à la vie adulte dans les forêts sacrées). En Guinée, il existe au sein des différentes structures traditionnelles, des pratiques culturelles qui ont, par le passé, contribué activement au maintien de la biodiversité des écosystèmes dont elles sont tributaires. Aujourd'hui, la réhabilitation de telles pratiques s'inscrit dans le cadre de la stratégie nationale pour la conservation et l'utilisation durable de la diversité biologique et le partage équitable des bénéfices de l'exploitation des ressources naturelles. Cette manœuvre s'appuie entre autres sur la prise en compte des modes traditionnels de gestion des expédients naturelles.

L'enjeu fondamental est celui-ci : comment les connaissances, innovations et pratiques traditionnelles en matière de biodiversité peuvent-elles être perpétuées dans un monde en métissage croissant, et dans quelle mesure peuvent-elles être utilisées et valorisées ? Cette question induit d'emblée le caractère pour le moins nuancé et complexe de la problématique. D'un côté, si ces savoirs et pratiques doivent être largement diffusés, les communautés doivent également pouvoir en garder le contrôle. De l'autre, si l'intérêt que revêtent ces savoirs peut permettre aux communautés de s'intégrer à la société moderne, il existe un risque sérieux de réification de ces derniers, et le fait de leur conférer une valeur économique n'entraîne pas automatiquement voire peut empêcher - une gestion durable in situ de la biodiversité.

\section{CADRE METHODOLOGIQUE}

\subsection{Zone d'étude}

Le lac Lungwe long d'approximativement $1.700 \mathrm{~m}$ et d'une largeur d'environ $700 \mathrm{~m}$ à une superficie de 1.093 $\mathrm{km} 2$ et située dans la partie extrême septentrionale de la Reserve Naturelle d'Itombwe (RNI) à $3^{\circ} 0^{\prime} 39^{\prime \prime}$ latitude Sud et $28^{\circ} 50^{\prime} 20^{\prime \prime}$ longitude Est à une altitude du niveau du lac de $2.786 \mathrm{~m}$ (Fig. 1 \&Fig 2). Avec une superficie de $5732 \mathrm{~km}^{2}$ de par ses énoncés des limites (Province du Sud Kivu, 2016), la Réserve figure parmi, diverses zones humides d'altitude du massif forestier d'Itombwe (Defailly, 2010). Sur le plan administratif, le lac 
Lungwe est situé à l'ouest de la plaine de la Ruzizi, à la latitude du village de Lemera. Il est situé dans la localité de Kigogo, chefferie de Luindi en territoire de Mwenga (Sud Kivu). Le lac occupe le fond d'une dépression bordée à l'Ouest et à l'Est par deux chaînes de collines atteignant 2.900 à $3.000 \mathrm{~m}$ dont la majeure partie se compose d'un socle ancien, ou bouclier, d'origine précambrienne, à partir duquel se sont formés les reliefs actuels. Les flancs de ces collines, non peuplés, sont couverts d 'une épaisse forêt de bambous, mélangés vers le bas d 'arbres feuillus parmi lesquels des Hageniaabyssinica et des Philippia. La crête des collines au contraire est partiellement dénudée et occupée par un Ericetum ou bien, là où les feux passent régulièrement, par une prairie basse (Marlier et al. 1955).

La dépression se prolonge au nord et au sud du lac; au Nord par une tourbière à Sphagnum et Ericacées, d'une étendue comparable à celle du lac entier ; au Sud s'étend également un marais àSphagnum beaucoup plus sec que la tourbière du Nord, déjà occupé par des arbustes et des graminées, indiquant un âge écologique plus avancé. Le lac est séparé de ce marais par une sorte de digue, d'une cinquantaine de mètres d'altitude, traversant la dépression de part en part. Le marais du Nord ne semble pas avoir d'autre écoulement par hautes eaux que le lac. Le marais du Sud communique au Sud-Ouest avec une vallée descendant vers un affluent de la rivière Muhembeji (Fig 1), sous-affluent de l'Ulindi. Dans la chaîne bordant le lac à l'Ouest se voit une interruption permettant le passage d'un petit affluent du lac constituant la Muhembeji elle-même.

Enfin, au nord du marais septentrional, une nouvelle digue transversale le sépare d'un grand marais, le Kilungutwe, qui constitue la source d'une petite rivière, la Kilungutwe qui, elle aussi, se réunit plus bas avec la Muhembeji et s'écoule ensuite par la Kadubu dans l'Ulindi à quelque $30 \mathrm{~km}$ au Nord-Ouest (à vol d'oiseau). Si l'on franchit la crête située à l'est du Lungwe, on redescend bientôt dans une vallée qui est la source de l'Ulindi supérieure qui, coulant vers le Sud, décrit une immense boucle pour repartir vers l'Ouest et le Nord-Ouest. De cette façon, l'Ulindi englobe dans son cours supérieur toute la dépression du Lungwe. Celle-ci est donc une portion du bassin supérieur de l'Ulindi, mais ses eaux n 'atteignent cette rivière par la Mwembeji que beaucoup plus loin en aval (Marlier et al. 1955).

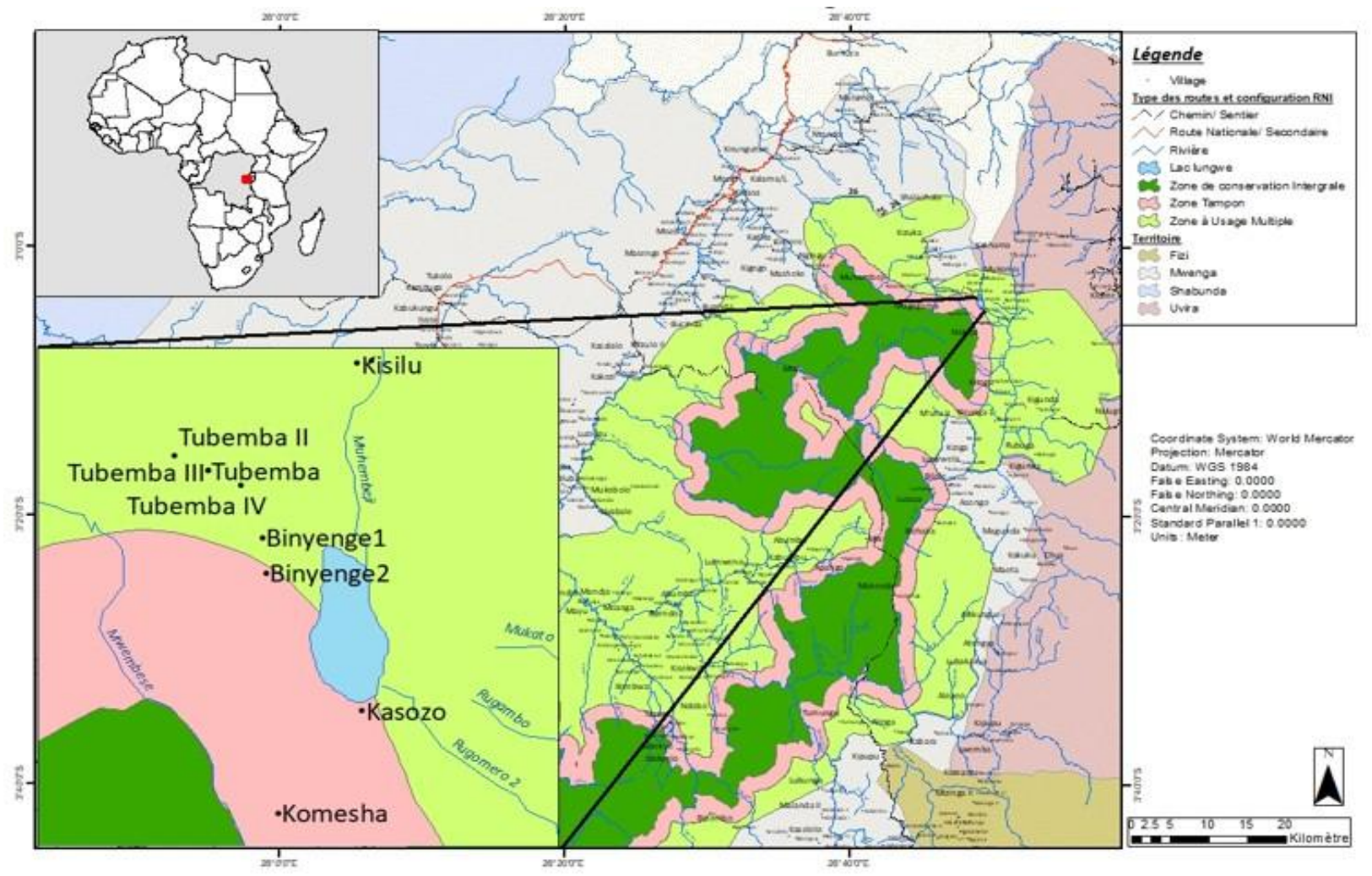

Fig 1. Localisation du lac Lungwe et villages enquêtés dans la RNI.

La présente étude a été effectuée au nord de la RNI à Muhuzi, l'une des localités se trouvant dans le groupement de Kigogo en chefferie de Lwindi dans territoire de Mwenga. Le choix porté sur cette entité se justifie par sa 
proximité avec le lac lungwe. Trois villages ont été enquêtés, à savoir : le village Binyenge, Lunvi et Kasozo (Fig 1) ; tous, habités en majorité par les Nyindu.

C'est entre les villages Binyenge et Kasozo à 2.786m d'altitude (Fig1) du bassin versant des collines Mukono et Kisilu, que s'observe un vaste marais d'eau stagnante en assèchement progressif à forme d'un calebassier appelé lac Lungwe. Le lac lungwe est le déversoir des plusieurs cours d'eaux dont le plus important c'est la rivière Muhembeji (Fig 1) qui l'alimente à partir du Nord et se déverse vers le Sud-ouest.

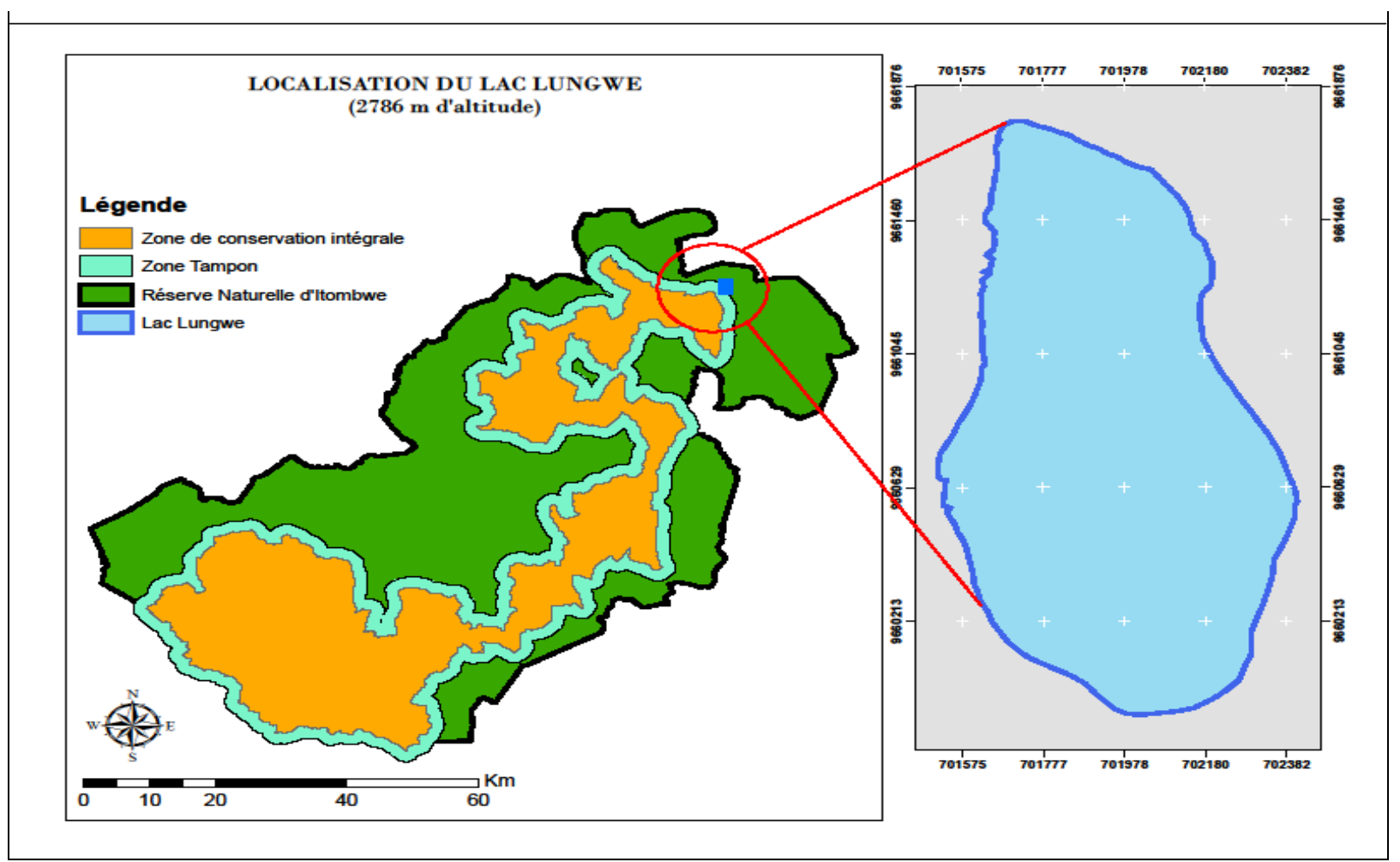

Fig 2. Localisation du lac Lungween gros plan dans la Réserve Naturelle d'Itombwe

Partant de la figure 2, il s'observe que le Lac Lungwe est localisé dans la partie Nord-est de la RNI à cheval entre la zone à usage multiple et la zone tampon.

\subsection{Question de recherche et objectif de l'étude}

Comme l'avait reconnu (Aubertin, 2006), parmi les fonctions sociales des chercheurs figure celle de produire des connaissances. Une connaissance orientée surtout vers des actions concrètes en insistant sur la nécessité d'approche, des concepts ou d'indicateurs utilisés. La présente étude focalise sur l'apport de la croyance de la population locale face à la protection du lac Lungwe. Dès lors. Comment agir pour que la RNI abritant le lac Lungwe ne soit pas perçu par la communauté comme "un espace stérile" (Sournia, 1990) soustrait à l'exploitation et sans rentabilité économique?

C'est à ce niveau qu'il sied de cerner l'importance d'analyser lesdites pratiques chez les peuples riverains du lac Lungwe en vue d'orienter leur intégration dans les politiques publiques relatives à la conservation de la nature. Cette étude vise ainsi d'une part à répertorier les savoirs et pratiques traditionnels de conservation de la nature existant chez les Lega, et d'autre part, à analyser l'impact des mutations socioculturelles sur leur pérennisation. Comment les peuples Lega riverains du lac Lungwe ont-ils conservé la nature dans leurs pratiques traditionnelles? Quels sont les facteurs influençant le déclin desdites pratiques? Telles sont les questions autour desquelles gravite cette étude. 
L'objectif global de cet article est de montrer dans quelles mesures les connaissances traditionnelles peuvent contribuer à la conservation des éléments de la biodiversité, à travers l'identification et l'analyse des connaissances et pratiques traditionnelles de conservation des éléments de la biodiversité des communautés locales riveraines du lac Lungwe. À terme, les éléments issus d'une telle synthèse devraient permettre d'intégrer pleinement les considérations socioculturelles et économiques ainsi que les questions de l'environnement, afin d'obtenir une plus large participation des différents acteurs. Le tout visant la construction d'une théorie des stratégies de conservation et de gestion sur lesquels reposent les décisions des acteurs impliqués.

\subsection{Méthodologie}

\subsubsection{Matériel et approche méthodologique}

L'enquête conduite en 2019 auprès de la population locale a été construite dans une perspective compréhensive et qualitative. Le choix s'est effectué selon la méthode de l'échantillon en cascade qui vise à questionner des personnes aux caractéristiques hétérogènes appartenant à un groupe « homogène » (Pires, 1997). Le séjour sur le site des enquêtés a permis les prises de contact individuelles. Les entretiens semi directifs menés en face à face se sont déroulés sur les lieux de travail,

\subsubsection{Matériels utilisés}

Les matériels utilisés pour la réalisation de cette étude sont, entre autres, le GPS modèle Map64S, un appareil photo numérique, un questionnaire d'enquête, un carnet de terrain et le stylo à bille et crayon.

\subsubsection{Méthodes de travail}

La taille de l'échantillon de cette étude était de 162 chefs de ménages paysans issus d'une population de 1072 ménages dans le groupement de Kigogo (Territoire de Mwenga, 2020). La table de TAKTO, pour P = 0.5 et un niveau de confiance de $95 \%$ donc $\mathrm{t}=1.96$ de précision et une marge d'erreur de 0.05 , donne l'effectif de l'échantillon de 147 ménages. La marge de 10\% a été majorée pour essayer de corriger les éventuels cas de non réponse. C'est pourquoi l'échantillon retenu a été de 162 chefs de ménages. Cet échantillon a été réparti en fonction des effectifs des ménages par village enquêté. Ainsi, il y a 49 personnes dans le village de Binyenge (30,25\%), 43 à Kasozo (26,54\%) et 70 à Lunvi (43,21\%) retenus pour cette étude. Dans l'échantillon total de 162 personnes, les hommes qui ont participé à l'étude représente 63,58\% (soit 103 hommes) et les femmes représentent $36,42 \%$ (soit 59 femmes) ; leur âge oscille entre 35 et 70 ans.

Pour de raison de bonne communication avec l'enquêté(e), les questions étaient traduites en Kiswahili, langue locale de la région et en cas de nécessité en dialectes locales pour certaines questions selon les différents villages enquêtés. C'est ainsi que le Kirega et le Kinyindu ont été utilisés selon la spécificité du milieu. Pour les centres les plus reculés et surtout pour les individus plus âgées, les conversations se sont faits, dans la plupart des cas, en existence de trois individus : l'enquêté, l'interprète et l'enquêteur dans un climat d'intimité et d'assurance que les données sont confidentielles et que l'anonymat sera respecté.

La méthodologie adoptée consistait à échanger avec les communautés locales réunies en focus groups tout en respectant la représentativité féminine. Un questionnaire orientait le débat et les réponses étaient notées. Pour celles nécessitant plus d'éclaircissements, la réponse du chef de village est celle qui était prise en considération. Une descente autour du lac Lungwe a été faite par les membres d'équipe et les envoyés spéciaux des chefs de deux villages. Avec un GPS allumé, un contour complet a été effectué à $\pm 2 m$ autour du lac Lungwe avec une erreur approximative inférieure ou égale à 3m. Les données d'enquête ont été traitées dans Excel 2007, et les coordonnées géographiques ont été traitées par ArcGIS 10.1. Les données brutes récoltées étaient encodées en Excel 2007. Les fréquences d'occurrence des déclarations des enquêtés ont été calculées et pour mesurer la force de la relation entre les impacts et les stratégies envisagées, le modèle généralisé linéaire a été utilisé pour déterminer l'influence des connaissances des impacts du changement par rapport aux savoirs et pratiques traditionnels sur le choix et l'adoption des stratégies de conservation. 


\section{RESULTATS ET DISCUSSION}

Répartition des enquêtés

Tableau 1. Répartition des enquêtés

\begin{tabular}{|l|l|l|l|l|l|}
\hline Village enquêté & Coordonnées géographiques du village & Homme & Femme & Total & $\%$ \\
\hline Binyenge & $0701068 / 9661402 / 2926 \mathrm{~m}$ & 31 & 18 & 49 & $30,25 \%$ \\
\hline Kasozo & $0702122 / 6960753 / 2916 \mathrm{~m}$ & 23 & 20 & 43 & $26,54 \%$ \\
\hline Lunvi & $0702517 / 9665370 / 2906 \mathrm{~m}$ & 49 & 21 & 70 & $43,21 \%$ \\
\hline Total & 103 & 59 & 162 & $100,00 \%$ \\
\hline
\end{tabular}

Il ressort du tableau 1 sur la répartition des enquêtés que l'échantillon de 162 personnes se caractérise par une diversité d'appartenance (âge, village, type d'activité), assurant un large spectre de réponses et un accès aux différents concepts existantes au sein du groupe professionnel. Les 162 personnes rencontrées sont âgées de 30 à plus de 65 ans pour tenir compte de la mémoire historique des faits. Ainsi, leurs témoignages reposent sur une pratique régulière et ancienne.

Origine du mot Lungwe

Les enquêtés révèlent à $98 \%$ que l'origine du mot «Lungwe » tire son origine d'un adage local de la tribu Nyindu «Bwami-bwa-Lungwe», par traduction littérale le mot Lungwe veut dire «Pouvoir, ou royaume ». Ce pouvoir, fait allusion à une considération mythique accordée aux eaux de ce lac par les autochtones. En effet, le lac Lungwe(Fig 3) est considéré comme un endroit un peu mythique suite à la force des pouvoirs coutumiers des chefferies riveraines (Defailly, 2010). Comme l'a reconnu (Claude, 1979) dans son étude sur l'historicité des mythes, tout adage paraît mystérieux tant qu'on ne connaît pas l'objet auquel il se rapporte. D'où, pour comprendre une culture et la portée des symboles qui l'accompagnent dans l'ensemble d'activités d'une société (Aubertin, 2006) souligne sur l'importance de comprendre au préalable les bases historiques et matérielles sur lesquelles s'édifie cette société.

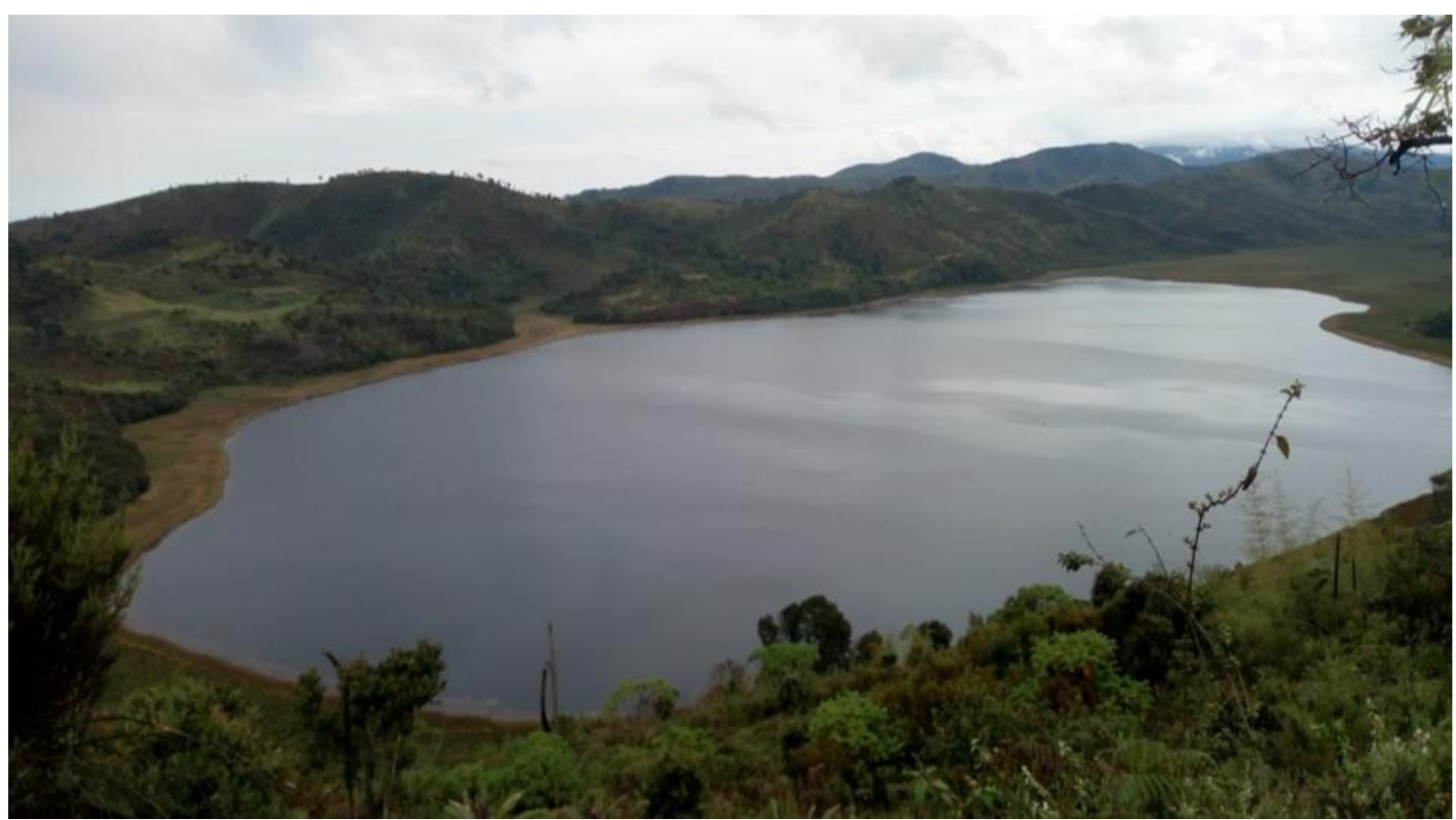

Fig3. Vue panoramique du lac Lungwe à partir du village Binyenge (Igunzi, 2019). 
Un mythe selon (Triplet, 2019), est un élément d'un mode de pensée fondamental d'une société communautaire. Les enquêtes ont confirmé à $95 \%$ que plusieurs mythes ${ }^{1}$ étaient jadis attribués au lac Lungwe par des ancêtres sous la forme d'une tradition. Bien que ces mythes n'aient pas fait l'objet d'une source écrite, les enquêtés reconnaissent qu'ils continuent à être transmis d'une génération à l'autre tout en souffrant actuellement des différentes interprétations d'une personne à une autre. La tradition à son tour telle que conçue par (Pascal, 1986)) est un ensemble de pratiques, d'actes ou d'énoncés mais aussi, comme une propriété commune aux actes et énoncés transmis d'une génération à l'autre et par laquelle certains membres de la communauté y pensent trouver une série de vérités fondamentales sur le monde. Toutefois, de la panoplie de mythes enregistrés autour du lac Lungwe dans la localité de Muhuzi, il s'avère que quelques-uns restent de stricte observance en ces jours par la population locale de la chefferie de Lwindi. Les enquêtés ont attribué un score à chaque croyances à savoir : l'Interdiction des doigter le lac Lungwe (75,8\%), l'interdiction de se baigner dans le lac Lungwe (94,7\%), l'interdiction d'utiliser le lac Lungwe comme lieu d'aisance (99,2\%), l'interdiction de se rapprocher ou circuler autour du lac Lungwe sans raison valable (71,6\%), 1'interdiction de chasser des petits gibiers autour du lac Lungwe (63,8\%), l'interdiction d'utiliser un chien de chasse autour du lac $(69,4 \%)$, l'interdiction d'abattre les arbres autour du lac Lungwe (64,3\%), et enfin l'interdiction de polluer une rivière se déversant dans le lac Lungwe (72,8\%).

L'état des lieux de leurs connaissances vise ainsi leur reconnaissance et leur valorisation, voire une gratification, sous la forme de «paiements de services écoenvironnementaux » de quatre types de fonctions : la position occupée par le lac dans la coutume, la permanence de sa présence, l'humidité élevée expliquant l'existence d 'une riche végétation d 'épiphytes sur les troncs des bambous et des bruyères arborescentes constituent des conditions idéales pour collecter des données essentielles sur l'état du milieu et son évolution.

Les mythes et les interdits autour du lac Lungwe ont évolué dans le temps faisant en ce jour l'objet d'une norme encrée dans les comportements, les mentalités et les cultures des certains autochtones. Le respect de ces mythes avait permis au lac Lungwe de conserver sa beauté actuelle, la propreté de ses eaux et de son paysage en certains endroits. Les enquêtés affirment à $76 \%$ qu'une forte pluie, généralement accompagnée de neiges, s'abattait dans les temps anciens à quiconque passait outre ses mythes. Ce qui faisait croire à une sanction naturelle infligée au coupable par la divinité protectrice du lac Lungwe. Cette nouvelle croyance a créé une considération toute particulière vis-à-vis des communautés vivant autour du lac Lungwe si bien que certains font actuellement du lac Lungwe leur lieu par excellence d'exécution de certains rites traditionnels. C'est le cas de la communauté réunie autour d'une secte dénommée «Mwanga-wa-mababu» (Lumière des ancêtres). En effet, selon les adeptes de cette communauté, le lac Lungwe, partant de son caractère mythique, son élévation en altitude, sa tranquillité et beauté de son paysage, sont là, des éléments de base de leur conviction qui témoignent que ce lieu, est un cadre idéal pour quiconque veut entrer en communion avec ce qu'ils qualifient « Dieux-des-ancêtres » A ce titre, en honorer d'un tel lieu, le port des habits tout blancs, l'allumage des bougies et la pulvérisation des parfums restent de stricte observance pendant toute la durée de leurs cérémonies culturelles autour du lac Lungwe. Concernant l'application de cette croyance locale face à la protection floristique du lac Lungwe, multiples croyances y sont rattachées en ces jours.

En plus de la croyance aux vertus curatives attribuées aux eaux du lac Lungwe par les autochtones, une seconde croyance est accordée à sa végétation composée en majorité des Cypéracées. En effet, plutôt qu'utiliser les plantes médicinales récoltées ailleurs dans la nature, les adeptes de la communauté « Mwanga-wa-mababu » et quelques communautés environnantes du lac, prônent sur l'utilisation des plantes médicinales poussant au bord du lac Lungwe qui seraient plus efficaces selon eux. Ainsi, 62\% des enquêtés affirment que les quelques reliques forestières actuellement visibles au Sud-est du lac lungwe, n'ont survécus que grâce au respect de cette croyance.

En effet, les rives du lac Lungwe(Fig 3) sont occupées par une végétation de tourbière Sphagnumsp., Erica, mais limitées au lac même par une ceinture assez épaisse (1-2 m) de Cyperussp., mêlés çà et là de touffes de Scirpus. Le fond du lac est abrupt dès les bords et, à la limite même de la ceinture des Cyperus, la profondeur est de 0,70 $\mathrm{m}$ à $1,70 \mathrm{~m}$. A ces profondeurs, s'enracine une ceinture de Potamogeton immergée, interrompue à de rares 
places là où la profondeur devient trop rapidement plus considérable. A ces Potamogeton, dans la direction de l'eau libre, fait suite une ceinture de Nymphéa à fleurs rose-mauve commençant à la profondeur de $1,50 \mathrm{~m}$ et s'étendant jusqu'à celle de $5 \mathrm{~m}$ environ. Elle a en général une largeur de 4 à $5 \mathrm{~m}$. Au-delà, aucune végétation ni immergée ni submergée ne se rencontre. Les rives proprement dites sont marécageuses et accéder à l'eau libre est extrêmement incommode, car de nombreux trous profonds de $0,5 \mathrm{~m}$ à $1 \mathrm{~m}$ remplis de Sphagnum barrent le passage. Ces caractéristiques combinées aux us et coutumes évoqués ci-haut sont de nature à renforcer la conservation du lac Lungwe et ses environs immédiats (Marlier et al. 1955).

Activités autour du lac Lungwe

Hormis les quelques activités agricoles a prédominance cultures de subsistance qui s'observent de nos jours en certains endroits autour du lac lungwe, aucune activité de grande envergure n'y a été documentée. Selon Carneiro da Cunha (2012), les savoirs locaux sont construits socialement et géographiquement et comportent une dimension historique dans la mesure où ils sont acquis par transmission. Les personnes enquêtées sont nées et ont grandi dans une famille (filiation) mais aussi dans la "communauté » (proximité), installée dans cette entité depuis des centaines d'années. Les connaissances sont acquises par l'observation et la reproduction de gestes, par l'écoute des récits autour du lac Lungwe et par le mythe répété des us et coutume lies à l'existence du lac Lungwe en tant que source incontestée du pouvoir. Cette croyance continue et constante crée une « intimité avec le milieu lacustre sacre » et permet de le découvrir. "Leurs savoirs sont basés sur l'expérimentation » canalisée et guidée par les aînés (Roué, 2012).

En effet, les savoirs naturalistes mis en évidence dans la section précédente peuvent être valorisés à travers la notion de services environnementaux (SEV). Froger et al. (2012) en proposent une typologie. La notion de « service-externalité » est la plus répandue pour évoquer l'impact non intentionnel mais réel de l'activité de l'homme sur les SES. L'embellissement, la assistance, l'entretien et l'aménagement du milieu naturel sont regardés comme des externalités évidentes. La seconde approche est celle du « service activité » mis en place et géré par les services publics (dans le domaine de l'eau par exemple). Cependant, la troisième approche qualifie le « service-produit joint » pour désigner les nouvelles filières de commerce équitable ou « bio » ne peut guère être envisagé dans la mesure où la faune du lac Lungwe est caractérisée par l'absence de poissons même dans les cuvettes les plus protégées. Dans les touffes de végétation, il a été impossible de déceler le moindre alevin. Ceci s'expliquerait par le fait que le lac Lungwe est le résidu d'une cuvette beaucoup plus étendue ayant fait partie du bassin de l'Ulindi vers laquelle il s'écoulait par le Sud et qu'à un stade ultérieur, tout le plateau s'assécha, faisant disparaître la faune aquatique et notamment les poissons. Il apparaît d'ailleurs que tout le haut plateau est complètement privé de poissons (Marlier et al. 1955).

Cependant, la faune inférieure est loin d 'être négligeable et la nourriture ne manquerait pas aux poissons éventuels. Il sied de mentionner que quelques minuscules colonies de Spongilles furent découvertes sur les plantes immergées en 1955 ainsi que de florissantes colonies d 'un magnifique bryozoaire sur toutes les tiges de Nymphéa qui représentent les seuls supports solides immergés, ainsi que d'innombrables Tubificides dans la vase du fond dont l'activité biologique a une action non négligeable sur la structure du sédiment (Marlier et al. 1955).

Le lac Lungwe ne possède pas non plus les larves de Tanytarsus caractéristique des lacs oligotrophes, c'est-àdire que l'oxygène n'est jamais fort abondant au fond. La raison en est probablement la richesse de l'eau en matières humiques. Par ailleurs, cette faune benthique, formée presque exclusivement de petits Crustacés, de Tubificides et de Tanypus est pauvre en espèces macroscopiques. Elle est, par contre, riche en individus ; il en est de meme pour le zooplancton du Lungwe qui est également pauvre en espèces mais les individus sont abondants (Marlier et al. 1955).

Caractéristiques géographiques du lac Lungwe

Le lac Lungwe a une forme ovale à grand axe Nord-Sud, long d'approximativement $1.700 \mathrm{~m}$; sa plus grande largeur, située peu au Sud de la ligne transversale médiane, est d'environ $700 \mathrm{~m}$. Ses rives sont occupées par une végétation de tourbière Sphagnum sp., Erica, mais limitées au lac même par une ceinture assez épaisse (1-2 m) de Cyperus sp., mêlés çà et là de touffes de Scirpus. Le fond du lac est abrupt dès les bords et, à la limite même de la ceinture des Cyperus, la profondeur est de $0,70 \mathrm{~m}$ à $1,70 \mathrm{~m}$. A ces profondeurs, s'enracine une ceinture de Potamogetonnageante, interrompue à de rares places là où la profondeur devient trop rapidement plus considérable. A ces Potamogeton, dans la direction de l'eau libre, fait suite une ceinture de Nymphéa à fleurs 
rose-mauve commençant à la profondeur de 1,50 m et s'étendant jusqu'à celle de $5 \mathrm{~m}$ environ. Elle a en général une largeur de 4 à $5 \mathrm{~m}$. Au-delà, aucune végétation ni nageante ni submergée ne se rencontre. Les rives proprement dites sont marécageuses et accéder à l'eau libre est extrêmement incommode, car de nombreux trous profonds de 0,5 m à $1 \mathrm{~m}$ remplis de Sphagnum barrent le passage (Marlier et al. 1955).

Le lac Lungwe, de par sa position au milieu d 'un marais très plat, passait pour une étendue susceptible de s'assécher périodiquement. En fait, c'est un véritable lac d'une profondeur appréciable. La plus grande profondeur mesurée a été de 19,60 m ; elle est située un peu au sud du milieu du lac. Des sondages équidistants, pratiqués le long de l'axe longitudinal, puis le long de l'axe transversal, indiquent l'existence d'un bassin unique à fond régulier incliné du Nord vers le Sud. La pente est en effet plus abrupte à la rive sud qu'à la rive nord.

Marlier et al. 1955 décrit le lac Lungwe comme le résidu d'une cuvette beaucoup plus étendue ayant fait partie du bassin de l'Ulindi vers laquelle il s'écoulait par le Sud. Sans doute, à cette époque lointaine recevait-il de l'eau par la rivière Luvubu, dont le cours est actuellement inversé et rejoint la vallée de la Ruzizi, et par le lit également inversé de l'actuelle Mwembeje. L'exutoire du lac Lungwe dit Mwembeje, à $500 \mathrm{~m}$ de sa sortie du lac, est un ruisseau de 10 à $15 \mathrm{~cm}$ de profondeur, d'eau claire et jaune à fond de quartz, abondamment garnie de plantes aquatiques. Près du lac, où l'eau ne coule guère, la végétation ripicole lacustre la borde. Là où elle se met à couler plus vite, la végétation devient rhéophile (présence d'un Nasturtium) ; une faune rhéophile s'y installe également : larves de Trichoptères Hydropsychides, Perlides, Éphémères des genres Baetis, Afronurus, Acentrella, etc., encore mêlée à des constituants de la faune lacustre. Ici non plus, aucun poisson ne se rencontre.L'eau du lac Lungwe, vue de haut, a une teinte brune, semblable à toutes les eaux de tourbières. Vue par transparence, sous une épaisseur de quelques $10 \mathrm{~cm}$, elle a une teinte champagne. Remarquablement claire, malgré sa couleur, l'eau du lac a une transparence de 2,60 m à midi (mesurée au disque de Secchi). Le pH à la surface était de 6,65; à $10 \mathrm{~m}$ de profondeur de 5,95 (pH mètre Beckman).

Tableau 2: Caractéristiques géographiques du lac Lungwe

\begin{tabular}{|l|l|l|l|}
\hline $\mathrm{N}^{\circ}$ & Caractéristiques géographiques du lac Lungwe & Valeur & EPE \\
\hline 01 & Altitude en mètre. & 2.786 & - \\
\hline 02 & Largeur du lac à la base en m & $874 \mathrm{~m}$ & - \\
\hline 03 & Latitude médiane du lac en UTM & 701936 & $2 \mathrm{~m}$ \\
\hline 04 & Longitude médiane du lac en UTM & 9660713 & $2 \mathrm{~m}$ \\
\hline 05 & Longueur du lac en mètre & $1.712,66$ & - \\
\hline 06 & Méridien central & 27,0000 & - \\
\hline 07 & Périmètre du lac en mètre & $4.684,5$ & - \\
\hline 08 & Superficie du lac en Km ${ }^{2}$ & 1,09282 & \\
\hline
\end{tabular}

Datum: WGS 1984 Zone 35S; False Easting 500 000,0000; False Northing10 000 000,0000

Projection : Transverse Mercator

Proposition de valorisation du lac Lungwe

$\mathrm{Au}$ vu que les croyances et mythes de la communauté locale vivant autour du lac Lungwe qui convergent vers la protection de sa biodiversité, l'on estime qu'il est encore grand temps de chercher des voies et moyens pour valoriser ces croyances d'une manière durable. En effet, la présence des communautés à proximité du lac Lungwe et son emplacement à cheval de la zone à usage multiple et la zone tampon de la RNI, est une opportunité à capitaliser en faisant du lac Lungwe un site touristique ou soit un site naturel sacré. Rappelons que la Déclaration de Rio sur l'environnement et le développement affirme que la meilleure façon de traiter les questions d'environnement, c'est d'assurer la participation de tous les citoyens concernés (Conférence des Nations Unies sur l'Environnement et le Développement, 1992). 
Lorsque des lieux sacrés se trouvent en dehors de toute aire protégée, dans la campagne, ou dans des paysages ruraux ou urbains, ils peuvent être mis en danger par tout un éventail de pressions qui vont du changement d'affectation des terres à la pauvreté en passant par l'urbanisation, des déplacements et la croissance démographiques, l'exploitation minière, agricole et forestière. Parmi les autres menaces, citons la surexploitation, des visites inappropriées, le pillage de lieux funéraires et de sites archéologiques, le vandalisme et la négligence (Wild \&McLeod, 2012).

Toutes les parties prenantes, particulièrement dans l'industrie touristique, doivent apprendre et partager les informations au sujet du tourisme et des sites naturels sacrés. Il est important, par exemple, que les brochures touristiques et le matériel d'information, tout comme les guides et les responsables touristiques, expliquent avec exactitude les valeurs culturelles locales pour permettre d'adopter un comportement approprié qui respecte les restrictions et les tabous. Citons, par exemple, le fait de savoir si l'on peut gravir ou pas une montagne sacrée, et avec quelle motivation, quels vêtements il faut porter et comment se comporter lorsqu'on entre dans un site sacré, et aussi quand il est interdit de photographier une cérémonie ou un rituel.

A ce titre les entités concernées par le lac Lungwe doivent impérativement être associés dans la définition commune et l'adoption des stratégies de valorisation du lac Lungwe. Deux options sont proposées à cet effet.

\subsubsection{Espace mythique Lac Lungwe comme lieu de randonnées touristiques}

Partant de cette option, il sera recommandé aux décideurs, d'orienter les objectifs de la conservation au développement des entités afin que ces communautés bénéficient des certains avantages pouvant en découler. A cette phase, (Mengue-Medou C. , 2002), recommande d'associer véritablement les populations locales à la prise des décisions dans la gestion d'une aire protégée, si l'on veut assurer un développement durable. Cette piste de valorisation du lac Lungwe s'avère réaliste aux contextes de gestion de la RNI entant qu'une aire protégée de catégorie VI de l'IUCN. Les AP de cette vocation, sont celles gérées et administrées principalement aux fins d'utilisation durable des écosystèmes naturels (Borrini-Feyerabend, 2014). Le paysage attrayant du lac Lungwe, après des aménagements des voies d'accès, lui prédispose à un site touristique par excellence pour des activités de visites lacustres programmées.

Le tourisme peut être une source alternative de revenus pour des communautés et, avec l'intérêt croissant pour la culture traditionnelle liée au site naturel sacré pouvant jouer un rôle en augmentant les revenus et les moyens de subsistance des communautés. L'écotourisme, qui met l'accent sur le support non seulement de la ressource mais aussi des communautés locales et aussi sur la promotion de visites respectueuses et à faible impact, serait le modèle idéal. S'il est pratiqué correctement, et géré selon un ensemble de principes éthiques, l'écotourisme visant les sites naturels sacrés peut profiter directement aux populations locales - économiquement, socialement et culturellement - à condition de respecter intégralement les systèmes de valeurs des populations locales et autochtones (Wild \&McLead, 2012).

Si les activités touristiques sont totalement gérées par les gardiens de leur communauté, qu'ils soient locaux, autochtones ou d'une religion dominante, il y a plus de chances pour que le tourisme soit profitable et accroisse les moyens de subsistance. Cela peut renforcer positivement la conservation des valeurs biologiques et culturelles des sites naturels sacrés. Par exemple, des études portant sur des forêts sacrées au Ghana ont montré que, lorsque les communautés gardiennes recevaient les bénéfices du tourisme et qu'elles les géraient ellesmêmes, la population locale était plus favorable à la conservation des singes en danger qui vivaient dans la forêt que les communautés d'une forêt sacrée où le tourisme était géré par le gouvernement, et qui ne recevaient que peu de bénéfices au niveau communautaire. (Ormsby, 2007).

Dès qu'un site intéresse des touristes, il faut souvent développer de nouvelles règles pour les appliquer aux visiteurs. Les réglementations récentes qui ont été générées dans de telles situations comprennent notamment celles concernant le comportement, le code vestimentaire et la photographie. Il est tout à fait normal de limiter la photographie à des endroits spécifiques, qu'ils soient sacrés ou profanes. Il est moins fréquent de limiter le genre de photos prises.

Bien géré, un tourisme responsable offre des possibilités de revenus économiques pour les communautés locales et autochtones, mais les activités touristiques doivent être adaptées, respectueuses et guidées par le système de 
valeurs des communautés gardiennes. Autant que possible, il faut supporter les entreprises de tourisme appartenant à et gérées par des communautés locales et autochtones, pour autant qu'elles fassent preuve de sensibilité environnementale et culturelle.

Ces considérations sur la valeur touristique justifient la réflexion sur le Payement des Services environnementaux (PSEV) avec deux objectifs essentiels : valoriser la connaissance des savoirs locaux des populations locales, assurer et promouvoir la conservation du milieu. L'approche avantageux institutionnaliste des PSEV offerte par Wunder (2005) les envisage comme une dédommagement de services rendus pour « inciter à articuler les décisions individuelles et collectives avec l'intérêt social de la gestion durable des ressources naturelles et la conservation de la biodiversité » (Froger et al. 2012), Par ailleurs, la compensation s'effectue dans « une logique d'additionnalité », le service écotouristique devant apporter un gain à la biodiversité et dans « une logique de conditionnalité » assurant l'effectivité du service, sa fourniture devenant obligatoire (Froger et al. 2012). L'activité devant être à la fois produite et utile à la biodiversité, le difficulté de l'évaluation de cette rendement reste entier,

Trois raisons essentielles liées à la problématique de la conservation de la biodiversité et de son caractère complexe, diffus et temporel méritent d'être pris en compte. En premier lieu, les SEV sont difficilement quantifiables. Comment évaluer l'impact de la participation des communautés locales aux instance délibératives de gestion de la RNI sur la protection de l'écosystème lacustre du lac Lungwe ? À quelle échéance, les actions d'éducation menées en direction des jeunes générations porteront elles leur fruit ? L'efficience à court terme des services fournis ne peut porter que sur leur effectivité et sur un rapport coût/avantage sur la base d'un scénario minimaliste (Pascual et al. 2010). En second lieu, les communautés locales ne sont pas toutes égales. Les activités touristiques ont le vent en poupe alors que la situation économique des communautés est vulnérabilisée par la baisse des ressources, le manque d'organisation en structure de gouvernance locale, des charges sociales considérées comme excessives. Le processus de PSEV suppose une réflexion en termes d'équité sur les montants et les destinateurs de la compensation entre la RNI et les communautés locales.

\subsubsection{Lac Lungwe comme site naturel sacré}

Dudley et al. (2005) ont trouvé des liens entre des coups et la conservation d'étendues telluriens et aquacoles dans tous les systèmes de croyance qu'ils ont étudiés. Un site naturel sacré tel que définie par (BorriniFeyerabend, 2014) est une zone terrestre ou aquatique portant une signification spirituelle particulière aux communautés. Il est composé de caractéristiques naturelles souvent unique et peut avoir une grande importance dans la conservation de la nature et les valeurs culturelles lui associées. Il est souvent un lieu de contact avec le domaine spirituel.

« Ces lieux, les plus propices à l'inspiration et à la spiritualité, les plus indispensables à la survie des espèces et des écosystèmes, les plus vitaux pour la sauvegarde des ressources alimentaires, de l'air et de l'eau, les plus essentiels à la stabilisation du climat, uniques pour le patrimoine culturel et naturel, méritent, entre tous, que l'humanité les entoure d'un soin particulier ». (Wild \&McLeod, 2012)

En ce qui concerne le lac Lungwe, sa valorisation comme site de randonnée touristique ou site naturel sacré, quelques travaux d'avance s'imposent toujours. Il s'agit entre autres, des travaux de traçage des pistes d'accès et la construction d'infrastructures d'accueil en des lieux stratégiques. Ceci, afin de concrétiser ce souhait de la communauté locale en une réalité qui pourra booster le développement de leur entité. La quiétude des eaux du lac Lungwe, son paysage attrayant et le courant d'air venant des sommets des collines, pourront contribuer à attirer la curiosité des visiteurs. Rappelons que lorsqu'il est bien pratiqué, les activités touristiques peuvent contribuer directement aux objectifs de la CDB (Leung, 2019). Néanmoins, un tourisme mal géré peut causer des impacts négatifs sur la biodiversité de l'aire protégée et aux communautés. A cet effet, l'analyse approfondie des plusieurs paramètres n'ayant pas fait objet de cette étude doivent entrer en ligne de compte dans la mise en œuvre de cette ambition de la communauté envoisinant le lac Lungwe. C'est le cas de l'étude de la concentration chimique des eaux du lac Lungwe, l'étude de sa profondeur ainsi que multiples essais pouvant conduire à une éventuelle adaptation des certaines espèces halieutiques dans le lac Lungwe, méritent d'être pris en considération pour la satisfaction des touristes. Les aires naturelles que des peuples tiennent pour sacrées se trouvent partout dans le monde. C'est un phénomène répandu que l'on observe dans presque tous les pays. L'on 
estime qu'il existe de nombreux milliers de systèmes de croyances différents autour du globe, et beaucoup d'entre eux ont une éthique en ce qui concerne la conservation

3.8 Diverses opportunités de réactivation des pratiques traditionnelles en étude

La tendance à l'effondrement des pratiques traditionnelles liées à la protection de la nature n'interdit pas de penser à leur réactualisation, eu égard aux enjeux environnementaux de l'heure. C'est sous cette perspective que Baco et al. (2007) notent que «les pratiques paysannes qui étaient jadis perçues comme catastrophiques et donc combattues sont actuellement acceptées et encouragées ». Au niveau local, la tradition n'a pas complètement disparu dans le chef de ces communautés. Elle est remémorée par ces dernières et oriente certaines de leurs activités (KyaleKoy\&Maindo, 2017). Le approche effectué entre conserve de la biodiversité et exploitation de savoirs traditionnels soulève de nombreuses interrogations sur l'utilisation même des savoirs, aussi bien du côté des décideurs que de celui des groupes sociaux ou individus associés à ce rapprochement (Redford et Mansour, 1996). Tantôt il est question de reconnaissance de droits de groupes sociaux et de singularité de leurs pratiques sociales, tantôt on se focalise sur la question du partage des avantages face à des projets de bioprospection

Dans le même ordre d'idées, Roussel (2005) démontre la durabilité des savoirs naturalistes locaux en soulignant « qu'appartenir à une tradition est considéré comme la garantie d'une certaine ancienneté et si les éléments de la biodiversité concernés sont parvenus jusqu'à nous, c'est que l'utilisation qui en est faite est nécessairement « durable»

\section{IMPLICATIONS POUR LA CONSERVATION ET CONCLUSION}

La conservation actuelle de la biodiversité du lac Lungwe comme résultats et apports d'une croyance locale de la communauté se marie avec l'avis de (Hanna, 1992) qui constatât qu'en Afrique, plusieurs espèces animales ou végétales, quelques fois des espaces forestiers étaient protégés par respect des traditions ancestrales. Bien que les croyances locales ayant qui concourent à la sauvegarde du lac Lungwe commencent à souffrir actuellement des petits dérapages par certaines personnes, nous estimons qu'il y a encore beaucoup des chances que cela marche une fois que la communauté y trouvera son compte. Rappelons que la protection des croyances et traditions locales reste un grand défi auquel se trouve confrontée la communauté internationale depuis une trentaine d'années (Mubalama, 2019). Le préambule de la Convention sur la Diversité Biologique, reconnait que les communautés locales dépendent traditionnellement des ressources biologiques sur lesquelles sont fondées leurs traditions (CDB, 1992). Depuis le sommet mondial sur le développement durable de 2002, la culture est désormais considérée comme une quatrième composante du développement durable. La culture, dans sa diversité, est une richesse. Il n'est donc plus possible de concevoir un développement durable qui ne respecterait pas la préservation des libertés et des droits culturels (Berger, 2014). C'est le cas du mont Athos en Grèce qui a été classé, en 1988, site du patrimoine mondial pour ses valeurs à la fois naturelles et culturelles (BorriniFeyerabend, 2014). Les caractères mythique, culturel et spirituel accordés au lac Lungwe, constituent donc une ressource non négligeable pour sa valorisation.

La réhabilitation des savoirs et savoir-faire sur la nature a une double conséquence : leur changement de statut et leur instrumentalisation politique par les communautés locales. D'outils d'utilisation durable et de gestion, ils sont de plus en plus souvent considérés à leur tour comme des objets de conservation, parties intégrantes de patrimoines qu'il s'agit dès lors de reconnaître, de valoriser et surtout de sauvegarder au même titre que les éléments de la biodiversité qui leur servent de support6. Les discours les plus fréquents insistent en effet sur l'érosion des savoirs face à la mondialisation et le risque de les voir disparaitre, en particulier dans les contextes de dégradation de l'environnement, de déforestation ou d'érosion de la biodiversité. Ces préoccupations, qui ne concernaient jusqu'alors que les éléments de la diversité biologique, impliquent maintenant la recherche d'informations et la mise au point d'instruments institutionnels et juridiques permettant de conserver et de valoriser les connaissances traditionnelles sur la nature et de renforcer les liens entre les communautés et leurs pratiques.

Le lac lungwe, entant qu'n petit lac d'altitude d'une superficie évaluée à $1,09 \mathrm{Km}^{2}$ (Tableau 2) situé dans la RNI, est considéré comme un lac mythique par les communautés locales. Une ferme croyance du mysticisme autours du lac Lungwe s'est enracinée dans le vécu quotidien des générations en générations et continue à avoir ses 
effets de nos jours quant à la manière de considérer et traiter la biodiversité du lac Lungwe. En effet, plusieurs interdits continuent à être attribués aux eaux du lac Lungwe et sa biodiversité par les communautés locales. Avec le temps, l'éveil de conscience et l'appropriation de la nouvelle approche de la conservation communautaires par les communautés, deux propositions pour la valorisation du lac Lungwe s'avèrent réalistes par rapport au contexte de gestion de la RNI, entant qu'aire protégée qui l'englobe. En effet, faire du lac Lungwe un site naturel sacré ou un site de randonnée lacustre lui ouvrira au grand public à la satisfaction des communautés qui l'entourent. Si les ancêtres se sont appuyés aux croyances, mythes et traditions pour protéger la biodiversité du lac Lungwe, les communautés enquêtées croient aux retombées d'activités touristiques pour le développement et le désenclavement de leurs entités. Une nette collaboration et transparence est nécessaire pour cette phase pour que cette activité soit bénéfique aux communautés. Durant les enquêtes, les communautés ont signalé l'inexistence d'espèces halieutiques dans le lac Lungwe en accusant la faible température des eaux comme cause majeure. Cette hypothèse n'a pas fait objet de la présente étude. Mais, mérite d'être approfondies par d'autres recherches. Le lac Lungwe entant que tel, reste est une potentialité qui mérite d'être valorisé sous toutes ses formes. Non seulement par les communautés locales qui l'entourent, mais surtout par les gestionnaires de la RNI, une aire protégée qui l'englobe.

La mise en œuvre d'une gestion durable des ressources lacustre suppose le développement de systèmes concrets de gratification assurant la reconnaissance et la valorisation de ces savoirs et des acteurs qui les détiennent. Ces mécanismes de compensation écotouristique doivent pouvoir prendre en compte deux types de services environnementaux rendus par les communautés. La première repose sur un engagement vis-à-vis de la protection du milieu lacustre et suppose un changement de pratiques. Le bénéficiaire direct est la biodiversité. Le second service consiste à cultiver les savoirs détenus en s'impliquant dans les actes de gestion durable des expédients, à court et moyen termes. Le bénéficiaire est la société (plus globalement l'humanité) et plus précisément les destinataires des services rendus que sont les acteurs institutionnels, les autres usagers du lac Lungwe et les populations locales. Dans tous les cas, ces gratifications doivent répondre à des besoins de justice et de répartition équitables.

Le recours aux connaissances traditionnelles peut notamment stimuler la coopération entre les gestionnaires des aires protégées et les communautés en créant le cadre d'une collaboration plus confiante. Les objectifs et les modalités d'organisation et de gestion de la conservation au travers l'écotourisme et l'éducation environnementale seront plus aisément compris par les populations riveraines du lac Lungwe pour autant que celles-ci comme partenaires, savent qu'elles en tireront un meilleur profit pour le présent et pour le long terme. Ce n'est que dans ce contexte que la RNI répondrait au mandat que le législateur reconnait à l'Institut Congolais pour la Conservation de la Nature (ICCN, 2018), notamment : (i) assurer la protection de la faune et de la flore ; (ii) valoriser la biodiversité en favorisant la recherche scientifique et en facilitant les activités d'écotourisme conformément à la législation en vigueur et dans le respect des principes fondamentaux de la conservation, et enfin (iii) réaliser ou de faire réaliser les études et d'en assurer la vulgarisation à des fins scientifiques, didactiques dans le domaine de la conservation.

Cet article révèle que les facteurs socioculturels tels que les traditions, les coutumes, les croyances et les tabous sont des éléments déterminants qui influencent les comportements des populations vis-à-vis de la sauvegarde de la biodiversité. Liés aux valeurs et normes sociales, ces facteurs motivent les décisions, pratiques et actions des populations. Les décideurs devraient logiquement valoriser les pratiques culturelles des communautés en matière de conservation de la biodiversité en préservant les traditions, les coutumes et les croyances des divers groupes culturels et en intégrant les acquis de la législation traditionnelle dans le cadre législatif et réglementaire étatique. Une piste forte captivante de production de ces acquis est la mise en œuvre de projets d'éducation relative à l'environnement dans les écoles. Lors des classes balades par exemple, un accent sera mis sur la spécial. Des différentes pratiques culturels (lieux sacrés, mares, législations coutumières) relatives à la conservation et la valorisation de la biodiversité tout en impliquant la communauté (implication du guérisseur traditionnel, du gestionnaire des forêts sacrées, des chefs coutumiers dans l'apprentissage des élèves). Des allants pratiques dans les milieux sacrés permettront aux élèves de se familiariser avec les espèces d'importance traditionnelle. Des recherches ainsi effectuées par les élèves pourront être partagées avec la population à travers des conférences débats, des théâtres scolaires et des ateliers de vulgarisation.

Les différents intérêts des connaissances et pratiques traditionnelles doivent conduire à les respecter, recenser, faire connaitre et utiliser en les valorisant aussi bien au profit des communautés locales qu'au profit de la 
conservation de la biodiversité de la RNI. Ceci permettrait de sauvegarder en même temps le patrimoine culturel qu'est le lac Lungwe.

\section{REFERENCES}

[1] Aubertin, C. (2006). Histoire et objectifs de l'Action transdépartementale incitative (ATI) « Aires protégées» de IRD 2004-2005-2006 , Florence Pinton et Estienne Rodary.

[2] Baco M.N., Biaou G., Pinton F. \& Lescure J.-P. (2007). « Les savoirs paysans traditionnels conservent-ils encore l'agrobiodiversité au Bénin ?», Biotechnol. Agron. Soc. Environ., Vol.11, n³, pp. 201-210.

[3] Bérard, L \& Marchenay, P. (2004). Les produits de terroir. Entre cultures et règlements. Paris, CNRS Éditions, 2004, 229 p.

[4] Berger, A. C. (2014). Le développement durable : retenir l'essentiel, Paris, Nathan, coll. « Repères pratiques ». .

[5] Berkes F. Colding J. \& Folke C. (2000). Rediscovery of Traditional Ecological Knowledge as Adaptive Management. Ecological Applications 10 (5) : 1251-1262.

[6] Berkes, F. (1993). Traditional ecological knowledge in perspective. Traditional Ecological Knowledge: Concepts and Cases, J.T. Inglis, Ed., Canadian Museum of Nature/International Development Research Centre, International Program on Traditional Ecological Knowledge International Development Research Centre, 1-9.

[7] Bonte, PI. (1992).(éds) Dictionnaire de l'ethnologie et de l'anthropologie Paris P.U.F. 1991 VIII-755.

[8] Borrini-Feyerabend, G. N. (2014). Gouvernance des aires protégées : de la compréhension à l'action. Collection des lignes directrices sur les meilleures pratiques pour les aires protegees.

[9] Byers, B. (1997). Démarche pour comprendre et influencer les comportements à l'égard de la conservation et de la gestion des ressources naturelles. Washington D.C. : Programme d'appui à la biodiversité, Bulletin sur la biodiversité africaine, numéro 4 .

[10] Carneiro da Cunha M. (2012). Savoirs autochtones : quelle nature, quels apports ? Leçon inaugurale au Collège de France prononcée le 22 mars.

[11] CDB. (1992). Convention sur la Diversité Biologique.

[12] Claude, M. (1979). Le mâle en gésine, ou de l'historicité des mythes. In: Cahiers d'études africaines, vol. 19, n73-76, Gens et paroles d'Afrique. Écrits pour Denise Paulme. $p 356$.

[13] Condé, K. (1997). Les organisations sociales traditionnelles et les services publics de conservation des écosystèmes dans le contexte guinéen (cas du Parc national du Haut Niger). Mémoire de diplôme d'études approfondies d'histoire, sociétés et civilisations, option anthropologie, Université de Rennes (France).

[14] Conférence des Nations Unies sur l'Environnement et le Développement. (1992). Déclaration de Rio sur l'environnement et le développement.

[15] Defailly, D. (2010). La foret d'Itombwe: Enjeux socio-economique et conservation de la nature en contexte congolais. Bukavu.

[16] Dove M.R. (2006). Indigenous People and Environmental Politics. Annual Review of Anthropology 35: 191-208.

[17] Dudley, N., Higgins-Zogib L. and Mansourian, S. (2005). "Beyond Belief, Linking faiths and protected areas to support biodiversity conservation". A research report by WWF, Equilibrium and The Alliance of Religions and Conservation (ARC). Available at: http://assets.panda.org/downloads/beyondbelief.pdf

[18] Ehinnou Koutchika R.I., Salako V.K., Tente B., Onodjè Agbani P., Chougourou D.C. 3, Boco Adjakpa J. \&Sinsin, B. (2014). «Étude écologique des bois sacrés des Communes de Glazoué, Savè et Ouessè au Bénin », Journal of Animal \&Plant Sciences, Vol.21, Issue 3, pp.3313-3323

[19] Froger G., Méral P., Le Coq J-F., Aznar O., Boisvert V., Caron A. \& Antona M. (2012). Regards croisés de l'économie sur les services écosystémiques et environnementaux. VertigO - la revue électronique en sciences de l'environnement 12 (3).

[20] Gouvrnorat de Province du Sud Kivu. (2016). Arrete provncialN ${ }^{\circ}$ 016/026/GP/SK/ du 26 juin 2016 portant $\mathrm{N}$ mesures provisoires d'actualisation des limites issues de la délimitation participative de la Réserve Naturelle d'Itombwe.

[21] Hanna. (1992). Optimal Credit Use with Uncertain Income" (Financial Counseling and Planning, Volume 3,1992).

[22] ICCN. (2018). Plan d'Aménagement et de Gestion 2018 - 2027, . 
[23] Kyale, K.J \& Maindo, MN. (2017). Pratiques Traditionnelles de Conservation de la Nature à L'épreuve des Faits Chez Les Peuples Riverains de la Réserve de Biosphère de Yangambi (RDC). European Scientific Journal March 2017 edition vol.13, No.8 ISSN : 1857 - 7881 (Print) e - ISSN 1857- 7431328

[24] Leung, Y.-F. (2019). Gestion du tourisme et des visiteurs dans les aires protégées : Lignes directrices pour la durabilité. Lignes directrices des meilleures pratiques dans les aires protégées $n^{\circ} 27$.

[25] Marlier, G., Bouillon, J., Dubois, J. Th \& Leleup, N. (1955). Le lac Lungwe. Académie Royale des Sciences Coloniales, Bulletin des Sciences. Nouvelle serie 1-1955-4.pp. 665-676.

[26] Mengue-Medou, C. (2002). Les aires protégées en Afrique : perspectives pour leur conservation, VertigO - la revue électronique en sciences de l'environnement [En ligne], Volume 3 Numéro 1 | avril 2002, mis en ligne le 01 avril 2002, consulté le 28 Avr 2020.

[27] Mubalama, L. (2019). Savoirs traditionnels conciliés aux connaissances scientifiques comme nouveau paradigme de la conservation des Aires Protégées : cas de Malambo (Réserve Naturelle d'Itom. Bukavu: Annales des Sciences et des Sciences Appliquées, Vol. 4(3/4, juillet 2018), 129-167.

[28] Ormsby, A. (2007). "Cultural and Conservation Values of Sacred Forests in Ghana". Paper presented at the Sacred Species and Sites meeting, Cambridge Centre for Landscape and People, 23-28 September, 2007, Cambridge, UK.

[29] Pascal, B. (1986). Tradition et vérité. In: L'Homme, 1986, tome 26 n97-98. L'anthropologie : état des lieux. pp. $309-329$.

[30] Pascual, U., Muradian R., Rodriguez L.C. \& Duraiappah A. (2010).Exploring the links between equity and efficiency in payments for environmental services: a conceptual approach. Ecological Economics 69 : 1237-1244.

[31] Pinto, F. et Grenand, P. (2007). Savoirs traditionnels, populations locales et ressources globalisées. In : Catherine Aubertin, Florence Pinto et Valérie Boisvert (dir). Les marchés de la biodiversité. IRD Editions. 165-194.

[32] Pires A. (1997). Échantillonnage et recherche qualitative : essai théorique et méthodologique. In Poupart, Deslauriers, Groulx, Laperrière, Mayer, Pires (Ed.) La recherche qualitative. Enjeux épistémologiques et méthodologiques. Montréal, Gaëtan Morin Éditeur : 113-169.

[33] Redford, K., and Mansour, J. (1996). Traditional Peoples and Biodiversity Conservation in Large Tropical Landscapes. The Nature Conservancy, Washington.

[34] Renard J., Henriot P., Schneider-Maunoury L. (2013). La gestion de la forêt de Mikea à Madagascar : comment concilier valeurs sociales, environnementales et économiques? Atelier les valeurs de l'environnement : entre éthique et économie, Paris, Ceres-Erti, $16 \mathrm{p}$.

[35] Roué M. (2012). Histoire et épistémologie des savoirs locaux et autochtones. Revue d'ethnoécologie 1, [En ligne], consulté le 23 avril 2020. URL : http://ethnoecologie.revues.org/813 ; DOI : 10.4000/ ethnoecologie.813

[36] Roué M. \& Nakashima D. (2002). Des savoirs «traditionnels » pour évaluer les impacts environnementaux du développement moderne et occidental. Revue internationale des sciences sociales 3-173:377-387.

[37] Roussel, B. (2005). "Savoirs locaux et conservation de la biodiversité : renforcer la représentation des communautés. », Mouvements, vol. 4, no 41, pp. 82-88.

[38] Savadogo, S., Ouédraogo, A\& Thiombiano, A. (2011). Diversité et enjeux de conservation des bois sacrés en société Mossi (Burkina Faso) face aux mutations socioculturelles actuelles. Int. J. Biol. Chem. Sci. 5: 1639-1658

[39] Sene C., Dioh P. \& Mormont, M. (2013). « Pratiques traditionnelles et gestion durable des ressources naturelles : Etude de cas des sites naturels sacrés côtiers et marins à Jaol-Fadiouth », ASRDLF - Appel à communication - Feuille de style, 18p.

[40] Sournia, G. (1990). Les aires de conservation en Afrique francophone : aujourd'hui et demain, espaces à protéger ou espace à partager ? Les cahiers d'Outre-Mer43(172). pp. 451-463.

[41] Sow, M. (2003) «Pratiques culturelles et conservation de la biodiversité en Guinée », Éducationrelative à l'environnement, Vol. 4, pp.289-295

[42] Territoire de Mwenga. 2020. Rapport trimestriel du territoire de Mwenga 2020 : Compilation des tableaux démographiques (20102020) de la population congolaise et étrangère du groupement de Kigogo dans la chefferie de Lwindi.

[43] Triplet, P. (2019). Dictionnaire encyclopédique de la diversité biologique et de la conservation de la nature .

[44] Tubiana, L. (2005). «Les savoirs naturalistes locaux, une question globale », Dans Bérard L., Cegarra, M., Djama., Louafi, S., Marchenay, P., Roussel, B \& Verdeaux F. (dir.) (2017). Biodiversité et savoirs naturalistes locaux en France, CIRAD, IDDRI, IFB, INRA, 
DOI: $10.51386 / 25815946 / \mathrm{ijsms}-\mathrm{V} 4 \mathrm{i} 2 \mathrm{p} 103$

Volume: 4 Issue: 2

March to April 2021

www.ijsmsjournal.org

[45] Wild, R \& McLeod, C. (Editors) (2012) Sites naturels sacrés : Lignes directrices pour les gestionnaires d'aires protégées. Gland, Suisse: UICN. xii + 108pp

[46] Wunder, S. (2005). Payments for environmental services: some nuts and bolts. CIFOR Occasional paper, $\mathrm{N}^{\circ} 42$. 